\title{
Arteriosclerose de Monckeberg como causa de isquemia crítica de membros inferiores: relato de caso
}

\author{
Monckeberg's arteriosclerosis as a cause of lower \\ limb critical ischemia: case report
}

\author{
Eduardo Lichtenfels ${ }^{1}$, Airton D. Frankini², Aline S. Becker ${ }^{3}$, Vinícius C. Pires ${ }^{3}$
}

\begin{abstract}
Resumo
A arteriosclerose de Monckeberg pode ser uma causa rara de isquemia grave dos membros inferiores. Relatamos o caso de um paciente com isquemia crítica do membro inferior, sem condições de revascularização, que evoluiu para amputação. $\mathrm{O}$ paciente não apresentava os fatores de risco clássicos para aterosclerose. $\mathrm{O}$ diagnóstico anatomopatológico foi de arteriosclerose por calcificação da média de Monckeberg.
\end{abstract}

Palavras-chave: Esclerose calcificante da média de Monckeberg, isquemia, membro inferior.

\section{Introdução}

A principal causa de isquemia de membros inferiores é o processo de aterosclerose, caracterizado pela formação de placas de ateroma nas artérias. A aterosclerose tem os seguintes fatores de risco principais: história familiar, sexo masculino, idade avançada, tabagismo, diabetes melito, dislipidemia e hipertensão arterial sistêmica ${ }^{1}$. Quando ocorre isquemia grave em pacientes que não apresentam nenhum dos fatores de risco mencionados, deve-se pensar em outras etiologias menos freqüentes e em processos distintos de aterosclero$\mathrm{se}^{1,2}$. O objetivo dos autores é apresentar um caso raro

1. Cirurgião vascular. Pós-graduando em Medicina, Fundação Faculdade Federal de Ciências Médicas de Porto Alegre (FFFCMPA), Porto Alegre, RS.

2. Professor adjunto, Disciplina de Cirurgia Vascular, FFFCMPA, Porto Alegre, RS. Cirurgião vascular e endovascular, Irmandade Santa Casa de Misericórdia de Porto Alegre (ISCMPA), Porto Alegre, RS. Membro titular, SBACV.

3. Médicos residentes, Angiologia e Cirurgia Vascular, FFFCMPA, Porto Alegre, RS.

Artigo submetido em 29.08.06, aceito em 23.01.07.

J Vasc Bras 2007;6(1):97-100.

Copyright $\odot 2007$ by Sociedade Brasileira de Angiologia e de Cirurgia Vascular.

\begin{abstract}
Monckeberg's arteriosclerosis can be a rare cause of severe lower limb ischemia. We report the case of a patient with critical lower limb ischemia without revascularization conditions progressing to $\operatorname{limb}$ amputation. The patient did not present any traditional risk factors associated with atherosclerosis. Histopathological diagnosis showed arteriosclerosis associated with Monckeberg's medial calcification.
\end{abstract} $\operatorname{limb}$.

Keywords: Monckeberg medial calcific sclerosis, ischemia, lower de isquemia crítica dos membros inferiores causada por arteriosclerose de Monckeberg.

\section{Descrição do caso}

Paciente de 64 anos, masculino, referia ser portador de asma brônquica com uso mensal de corticosteróide injetável nos últimos 6 meses. Negava tabagismo, diabetes melito, dislipidemia, etilismo ou hipertensão arterial sistêmica. Relatava ter notado, há cerca de 1 ano, o aparecimento de pequenas lesões necróticas nas extremidades dos pododáctilos à direita, não associadas à dor ou a outro sintoma local. Negava história de traumatismo. Referia tratamento apenas com curativos há 3 meses, sem resolução das lesões. Há cerca de 1 mês, passou a referir dor em repouso na extremidade.

Ao exame físico, apresentava membro inferior direito frio, edemaciado, com ausência de pulsos poplíteo e distal, palidez acentuada à elevação, hiperemia reativa moderada e tempo de enchimento venoso de 45 segundos. Havia presença de focos de necrose seca em ambos os pés, além de gangrena úmida do segundo pododác- 
tilo direito. O membro inferior esquerdo estava indolor, hiperemiado, com pulso femoral normal e ausência de pulsos poplíteo e distais.

Os exames laboratoriais apresentavam: leucocitose $(15.600 / \mu \mathrm{L})$ com desvio à esquerda $(2 \%)$, eosinofilia $(12 \%)$ e plaquetas em forma de agregados; $159 \mathrm{mg} / \mathrm{dL}$ de triglicerídeos; $129 \mathrm{mg} / \mathrm{dL}$ de LDL; $31 \mathrm{mg} / \mathrm{dL}$ de HDL; $192 \mathrm{mg} / \mathrm{dL}$ de colesterol total; $93 \mathrm{mg} / \mathrm{dL}$ de glicemia; e $1,5 \mathrm{mg} / \mathrm{dL}$ de creatinina. $\mathrm{O}$ fator reumatóide foi nãoreagente, o fator antinuclear foi não-reagente, crioglobulinas foram negativas, anticorpos anticardiolipina IgM e IgG foram negativos, anticorpo anticitoplasma foi não-reagente, sorologia para sífilis foi negativa $\mathrm{e}$ sorologias para vírus das hepatites $\mathrm{B}$ e $\mathrm{C}$ foram negativas. Os seguintes exames mostraram pequenas alterações: velocidade de sedimentação globular (VSG) de 65 $\mathrm{mm} / \mathrm{h}$; proteína C reativa de $99,7 \mathrm{mg} / \mathrm{L}$; fator $\mathrm{V}$ de Leiden de 62\%; complemento C3 de $206 \mathrm{mg} / \mathrm{dL}$; complemento C4 de $56 \mathrm{mg} / \mathrm{dL}$; e fibrinogênio de $503 \mathrm{mg} / \mathrm{dL}$. As provas de função hepática mostraram-se alteradas, com tempo de protrombina de 50\%, TGO de 115 $\mathrm{UK} / \mathrm{mL}$ e TGP de $75 \mathrm{UK} / \mathrm{mL}$. Os demais exames foram: antitrombina III de $0,22 \mathrm{~g} / \mathrm{L}$; proteína $\mathrm{C}$ de $52 \%$; proteína $\mathrm{S}$ de $79 \%$; e homocisteína de $7,5 \mu \mathrm{mol} / \mathrm{L}^{-1}$. O paciente foi submetido à arteriografia de membro inferior direito, que demonstrou múltiplas estenoses e irregularidades da artéria femoral superficial (Figura 1), artéria poplítea pérvia, doença difusa, múltiplas estenoses das artérias tibiais e fibular (Figura 2) e leito distal com calcificações difusas da parede arterial, sem enchimento com contraste (Figura 3). O paciente foi submetido à exploração da artéria poplítea supragenicular. O exame macroscópico demonstrou artérias endurecidas, com placas extensas e intenso processo de calcificação parietal, sendo que as artérias possuíam, à palpação, textura óssea. O sistema arterial não apresentava condições de pinçamento, arteriotomia ou revascularização. Decidiu-se, então, devido ao quadro de isquemia crítica, pela amputação transfemoral.

O paciente apresentou boa evolução pós-operatória. $\mathrm{O}$ exame anatomopatológico do membro amputado demonstrou necrose isquêmica, aterosclerose com placas fibrosas e calcificação da camada média em artérias

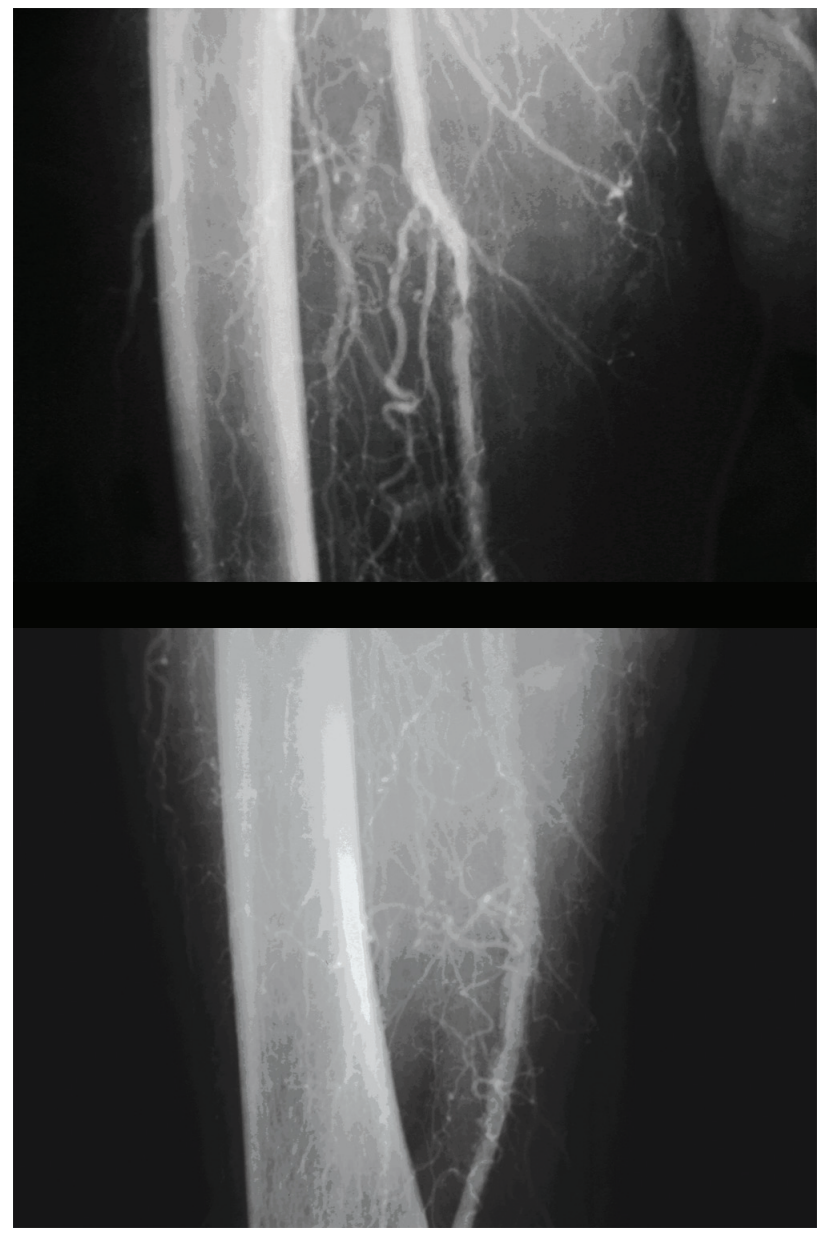

Figura 1 - Arteriografia evidenciando as múltiplas lesões e irregularidades da artéria femoral superficial

femoral, poplítea e tibiais. O diagnóstico final foi de calcificação da média de Monckeberg.

O membro inferior esquerdo foi avaliado através de eco-Doppler arterial, que revelou calcificações parietais importantes e oclusão das artérias tibiais anterior e posterior, fibular e pediosa. Porém, como o paciente não apresentava dor em repouso ou lesões significativas, optou-se pelo tratamento conservador.

\section{Discussão}

A calcificação da média de Monckeberg é uma condição mais prevalente em pessoas acima de 50 anos e do sexo masculino. Aparentemente, não está associada a outros fatores de risco, embora a incidência seja maior em pacientes diabéticos ${ }^{1,3,4}$. A prevalência na população, em estudo recente, foi de 13,3\% no sexo masculino 


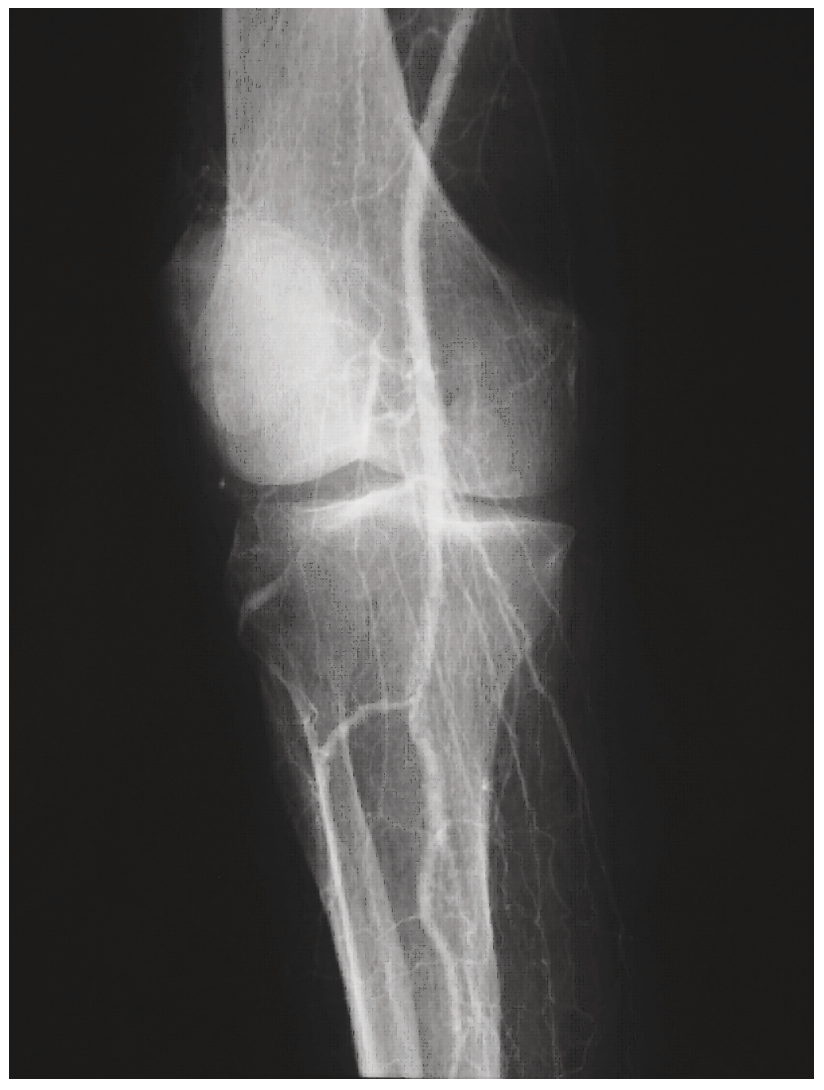

Figura 2 - Arteriografia evidenciando as lesões das artérias poplítea, tibial anterior e fibular

e de $6,9 \%$ no sexo feminino ${ }^{5}$. Essa é uma causa rara de arteriosclerose, na qual ocorrem depósitos de cálcio na camada média das artérias, podendo eventualmente evoluir para processo de ossificação ${ }^{2,4,6}$. Esses depósitos de cálcio podem adquirir a forma de placas irregulares da média ou discretos anéis transversais, criando nódulos. O processo de calcificação é, em alguns casos, perceptível em estudo radiográfico. Embora as lesões não costumem invadir a parede dos vasos, as artérias acometidas também podem desenvolver aterosclerose ${ }^{2}$. Sua etiologia permanece desconhecida ${ }^{4}$. Em nosso relato, o paciente tinha 64 anos, não era portador de diabetes melito e apresentava calcificações ao estudo angiográfico.

Lehto et al. ${ }^{7}$ demonstraram, em seu estudo, que a arteriosclerose de Monckeberg é um fator de risco independente para morbimortalidade por eventos cardiovasculares.

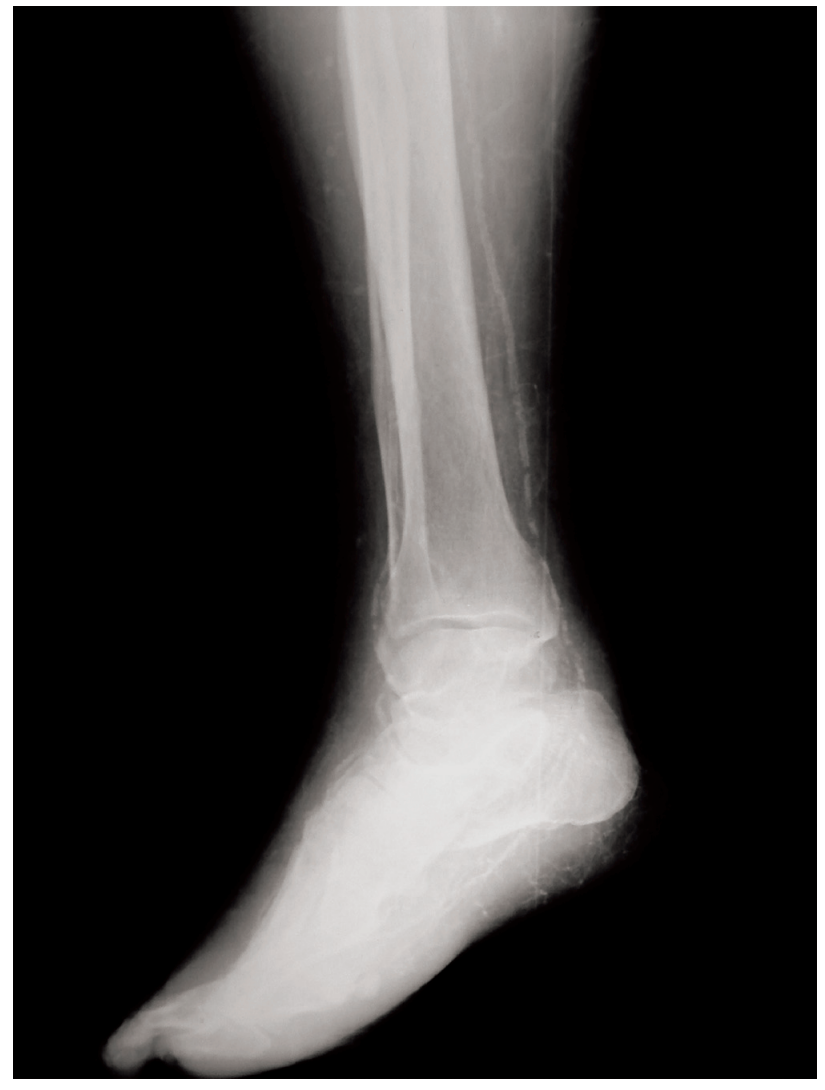

Figura 3 - Arteriografia mostrando o leito distal e a calcificação da artéria tibial posterior

Os poucos relatos na literatura referem-se a lesões em artérias dos membros inferiores e, com menor freqüência, em artérias viscerais. $\mathrm{O}$ acometimento das artérias coronarianas, periféricas e viscerais pela calcificação de Monckeberg foi descrito por Lachman et al. ${ }^{8}$, em 1977. Lanzer ${ }^{9}$ relatou o caso de arteriosclerose de Monckeberg envolvendo a aorta, as artérias pélvicas e de membros inferiores. Couri et al. ${ }^{10}$ relataram o acometimento não-vascular de tecidos moles (faringe e laringe) pela esclerose de Monckeberg.

Nosso relato apresenta um paciente com quadro de isquemia crítica que evoluiu desfavoravelmente para amputação primária, sem apresentar os fatores de risco tradicionais para o desenvolvimento de aterosclerose (tabagismo, diabetes melito, dislipidemia e hipertensão arterial). As artérias exploradas encontravam-se bastante comprometidas por processo de calcificação da parede, inviabilizando qualquer possibilidade de revascularização. Houve a formação de um processo de 
arteriosclerose pouco usual, distinto da aterosclerose tradicional, que, através do estudo anatomopatológico da peça de amputação, revelou tratar-se da calcificação da média de Monckeberg.

Salientamos que a arteriosclerose de Monckeberg não impede o processo de revascularização em todos os casos. Há situações nas quais é possível a confecção de anastomoses, evitando-se o pinçamento da artéria. Em outros casos, como no descrito, o comprometimento é tão difuso, espesso e calcificado que a arteriosclerose inviabiliza o pinçamento e a revascularização arterial, sendo determinante para a indicação da amputação primária $^{8,11}$. Shabat et al. relatam um caso de ruptura da artéria poplítea durante cirurgia ortopédica do joelho devido ao intenso processo de calcificação da

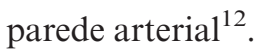

Concluímos que, nos casos de pacientes portadores de doença arterial obstrutiva periférica com etiologia não definida e ausência dos fatores de risco tradicionais, deve-se lembrar que a calcificação da média de Monckeberg, apesar de rara, pode responder pela vasculopatia periférica encontrada. São necessários mais estudos para um melhor entendimento dessa patologia bem como para a melhora do seu diagnóstico e do seu tratamento.

\section{Referências}

1. Sumpio BE. Aterosclerose: considerações biológicas e cirúrgicas. In: Ascher E, ed. Haimovici: cirurgia vascular. $5^{\mathrm{a}}$ ed. Rio de Janeiro: Revinter; 2006. p. 148-52.

2. Schoen FJ, Cotran RS. Blood vessels. In: Cotran RS, Kumar V, Collins T. Robbins: pathologic basis of disease. 6th ed. Philadelphia: WB Saunders; 1999. p. 493-542.
3. Okuno Y, Sato K. Diabetes mellitus and vascular calcification. Clin Calcium. 2002;12:1094-101.

4. Top C, Cankir Z, Silit E, Yildirim S, Danaci M. Monckeberg's sclerosis: an unusual presentation--a case report. Angiology. 2002;53:483-6.

5. Kroger K, Stang A, Kondratieva J, et al. Prevalence of peripheral arterial disease - results of the Heinz Nixdorf recall study. Eur J Epidemiol. 2006;21:279-85.

6. Vattikuti R, Towler DA. Osteogenic regulation of vascular calcification: an early perspective. Am J Physiol Endocrinol Metab. 2004;286:E686-96.

7. Lehto S, Niskanen L, Suhonen M, Rönnemaa T, Laakso M. Medial artery calcification. A neglected harbinger of cardiovascular complications in non-insulin-dependent diabetes mellitus. Arterioscler Thromb Vasc Biol. 1996;16:978-83.

8. Lachman AS, Spray TL, Kerwin DM, Shugoll GI, Roberts WC. Medial calcinosis of Monckeberg. A review of the problem and a description of a patient with involvement of peripheral, visceral and coronary arteries. Am J Med. 1977;63:615-22.

9. Lanzer P. Monckeberg media calcinosis. Z Kardiol. 1998;87:586-93.

10. Couri CE, da Silva GA, Martinez JA, Pereira Fde A, de Paula FJ. Monckeberg's sclerosis - is the artery the only target of calcification? BMC Cardiovasc Disord. 2005;5:34.

11. Fuessl HS, Schalzky H, Schewe S, Frey KW, Goebel FD. Pathogenesis and clinical significance of Monckeberg medial calcinosis. Klin Wochenschr. 1985;63:211-6.

12. Shabat S, Mann G, Stern A, Nyska M, Witz M. Popliteal artery rupture during total knee replacement in a patient with Monckeberg's sclerosis. Case report. J Knee Surg. 2004;17:117-9.

Correspondência:

Airton Delduque Frankini

Rua Quintino Bocaiúva, 1290/502

CEP 90440-050 - Porto Alegre, RS

Tel.: (51) 3332.2410/3222.2716

E-mail: frankini@terra.com.br 